\title{
A ORIGEM E EVOLUÇÃO DA FISIOTERAPIA: DA ANTIGUIDADE AO RECONHECIMENTO PROFISSIONAL
}

\author{
Robson Feliciano da Silva ${ }^{\mathrm{T}}$ \\ Sérgio William da Silva Santos ${ }^{2}$ \\ Adriele Sena dos Santos ${ }^{3}$ \\ José Luís dos Santos Filho ${ }^{4}$
}

RESUMO: A Fisioterapia tem sua fundamentação na antiguidade (foi Hipócrates, o pai da medicina que utiliza pela primeira vez o termo "Medicina de Reabilitação"), é uma profissão da área da saúde, considerada como campo de atuação profissional. Desde os primórdios, foi utilizada de forma empírica e no decorrer de sua história, acompanhou as mudanças e transformações do século XX. Foi na Europa, o surgimento das primeiras escolas, primeiramente na Alemanha e depois da Inglaterra. No Brasil com as leis, decretos e emendas conquistaram a legalidade profissional, conferindo direitos e deveres. Atualmente, o processo de formação do fisioterapeuta está direcionado a desenvolver competências e habilidades gerais para atenção à saúde, como ações de prevenção, promoção e proteção da saúde, além da reabilitação individual e coletiva. Esse trabalho teve como objetivo mostrar a evolução da fisioterapia considerando três grandes subdivisões da história da humanidade: períodos que antecede a Primeira Guerra mundial (Antiguidade, Idade Média, Renascimento e Industrialização), períodos das grandes guerras e período contemporâneo. A metodologia utilizada foi por meios de buscas a referências bibliográficas nas bases de dados eletrônicos. $\mathrm{O}$ critério de inclusão do estudo foi a abordagem de temas relacionados às questões a evolução da Fisioterapia e o reconhecimento profissional. Foram utilizados para a busca, artigos disponibilizados na íntegra em português e em inglês. Conclui que a fisioterapia, ao longo da história, vem fortificando o reconhecimento da importância da profissão, estabelecendo funções ao movimento corporal, mas também potencializando funções ao sujeito como pessoa. As transformações foram positivas, tanto no âmbito profissional, quanto no âmbito acadêmico. O fisioterapeuta está presente em todas as escalas com todas as áreas em crescimento.

Palavras-chave: Evolução. Fisioterapia. Reconhecimento Profissional.

\footnotetext{
I Especialista em Ensino de Biologia pela Universidade Cândido Mendes - UCAM. Especialista em Análises Clínicas pela Faculdade Escritor Osman da Costa Lins - FACOL. Graduado em Licenciatura em Ciências Biológicas pela Universidade Federal de Pernambuco - UFPE. Acadêmico em Fisioterapia pela UNIFACOL - Centro Universitário FACOL. E-mail: robsonf.silva@unifacoledu.br.

2 Acadêmico em Fisioterapia pela UNIFACOL - Centro Universitário FACOL. Email: swilliam.315@gmail.com

3 Acadêmica em Fisioterapia pela UNIFACOL - Centro Universitário FACOL. Email: adriele_o7@hotmail.com

Acadêmico em Fisioterapia pela UNIFACOL - Centro Universitário FACOL. Email: j.luisi@outlook.com.br.
} 
ABSTRACT: Physiotherapy has its foundation in antiquity (it was Hippocrates, the father of medicine who used for the first time the term "Rehabilitation Medicine"), it is a profession in the health field, considered as a field of professional activity. Since the beginning, it has been used empirically and throughout its history, it has followed the changes and transformations of the 2oth century. It was in Europe, the emergence of the first schools, first in Germany and then in England. In Brazil, with laws, decrees andamendments, they gained professional legality, conferring rights and duties. Currently, the physiotherapist training process is aimed at developing general skills and abilities for health care, such as prevention, health promotion and protection actions, in addition to individual and collective rehabilitation. This work aimed to show the evolution of physiotherapy considering three major subdivisions of human history: periods before the First World War (Antiquity, Middle Ages, Renaissance and Industrialization), periods of the great wars and contemporary period. The methodology used was through searches of bibliographic references in electronic databases. The study inclusion criterion was the approach of themes related to the issues of the evolution of Physiotherapy and professional recognition. Articles available in full in Portuguese and English were used for the search. It concludes that physiotherapy, throughout history, has been strengthening the recognition of the importance of the profession, establishing functions to body movement, but also enhancing functions to the subject as a person. The changes were positive, both professionally and academically. The physiotherapist is present at all scales with all areas growing.

Keywords: Evolution. Physiotherapy. Professional Recognition.

\section{INTRODUÇÃO}

O Conselho Federal de Fisioterapia e Terapia Ocupacional (COFFITO) na Resolução $\mathrm{n}^{\circ}$. 8o, de 9 de maio de 1987, apresenta a fisioterapia como sendo ciência aplicada, cujo objeto de estudos é o movimento humano, seja nas suas alterações patológicas, ou nas suas repercussões psíquicas e orgânicas, com objetivos de preservar, manter, desenvolver restaurar a integridade de órgãos, sistema ou função. A fisioterapia é a profissão da área da saúde cujo principal propósito é a promoção da saúde por meio da aplicação de princípios científicos para evitar, identificar, avaliar, corrigir ou melhorar a disfunção aguda ou prolongada dos movimentos (COFFITO; TONELINI, 2009).

A profissão surgiu a partir de vários conhecimentos e práticas, primeiramente dos conhecimentos das profissões de enfermagem, medicina e educação física. Cada uma dessas profissões agregou saberes que permanecem no cotidiano do fisioterapeuta na atualidade (ESPÍNDOLA e BORENSTEIN, 20II).

Para a compreensão dessa ciência é preciso conhecer sua história, que teve seus primórdios na antiguidade (4.00o a.C. e 395 d.C.). Em 2.698 a.C., aparecem na China os 
primeiros registros do uso de meios físicos com o desígnio terapêutico (no caso exclusivo da cinesioterapia), e logo depois, na Índia. Contudo, recursos físicos naturais (que são a base da fisioterapia utilizadas pelos povos antigos), como banhos de sol, são exibidos como tratamentos medicinais em toda antiguidade e desenvolveram-se como ciência e vem sendo utilizados até os dias atuais (GAVA, 2004 apud NASCIMENTO, 201I).

A ideia de usar meios físicos para curar e restaurar as funções do corpo foi desenvolvida ao longo de milhares de anos. A utilização da eletricidade, de exercícios, de massagem e de terapias manuais cresceu de forma sistemática no decorrer dos últimos trezentos anos, ganhando o reconhecimento dos clínicos e de toda a população. Ao longo de toda da história, a prática clínica foi dinâmica, adaptando-se às necessidades da sociedade na resolução das alterações do movimento e da função (FONSECA, 2012).

A Fisioterapia como profissão surge em meados do século $\mathrm{XX}$, as duas guerras mundiais deixaram um número elevado de pessoas com lesões graves e que necessitavam de uma abordagem de reabilitação para reinserira-las novamente em uma vida ativa. Assim, a Fisioterapia acompanhou as grandes transformações do século XX agregando novas descobertas e técnicas às suas práticas, desenvolvendo um campo específico de atuação, independente das outras áreas da saúde (CREFITO 3 ).

A importância da realização desse trabalho está na implicação da possível contribuição do entendimento da evolução da fisioterapia, fundamentando a sua história numa visão ética, critica e reflexiva, integralizando com a sociedade e o homem. $\mathrm{O}$ presente estudo teve como objetivo mostrar os aspectos históricos e legais da fisioterapia desde os primórdios, na antiguidade, até os dias atuais. Reconhecendo o profissional fisioterapeuta como parte integrante e necessária da equipe de saúde.

\section{METODOLOGIA}

O estudo foi por meio de buscas a referência bibliográfica nas bases de dados online, como Biblioteca Virtual de Saúde, o SciELO - Scientific Electronic Library Online e PubMed, acessados por meio da Biblioteca Científica Eletrônica online. Foram utilizados para a busca e critérios de inclusão, artigos em português e inglês. Foram revisados artigos, que, sob distintas óticas e objetivos descreveram sobre a evolução da Evolução, Fisioterapia e o Reconhecimento profissional. Após a avaliação dos resumos, os estudos que atenderam 
aos critérios de inclusão foram selecionados. O corpus gerado pelo levantamento bibliográfico consistiu alguns registros de produções, cujos dados foram organizados em um banco de dados, os quais foram analisados em diferentes recortes: por domínio e temática, tipos de veículos de divulgação, palavras-chave e conteúdo. Procurou-se, então, no conjunto das obras de referência o necessário para sua abrangência, de forma a poder compreender melhor a evolução da fisioterapia.

\section{A origem e Evolução}

Foi Hipócrates, o pai da medicina, (46o a 377 a.C.) que utilizou pela primeira vez o termo "Medicina de Reabilitação," caracterizando a fisioterapia em uma de suas áreas de atuação, hoje admite-se também que foi ele um dos primeiros a descrever e documentar tratamentos para a coluna vertebral, a qual não mudou muito até o final do século XIX. Em relação à reabilitação, encontram-se as primeiras referências, entre os séculos VI e II a. C, a utilização de próteses no mundo ocidental (KLADNY, 2015).

A fisioterapia associa-se no contexto histórico da medicina curativa. $\mathrm{Na}$ antiguidade a principal preocupação dos atuantes da saúde era a de curar. Os conhecedores dos métodos naturais, empregava-os como formas terapêuticas. A valorização do movimento humano e a cura por esse método estão registrados na literatura chinesa, praticadas desde o ano de 2698 a.C. Na Grécia Antiga, a atividade muscular e a reabilitação pelo movimento foram descritas pelo filósofo Aristóteles (384 a.C.) (FONSECA, 2012).

$\mathrm{Na}$ idade Média (século IV a XV) para a igreja o corpo humano era considerado sagrado, receptáculo da alma, e como tal, as pesquisas era quase que proibidas. A consolidação do cristianismo implicou um abandono da cultura da saúde. Essa exaltação da fé provocou uma interrupção no avanço dos estudos da área de saúde no geral. Para interesse das camadas privilegiadas, a nobreza e o clero, os exercícios passaram a ter outros objetivos eles eram utilizados unicamente como diversão ou aumento da potência corporal (BARROS, 2002 apud NASCIMENTO, 2011).

Após o período de estagnação dos estudos, surge o Renascimento (período entre os séculos XV e XVI), momento de desenvolvimento científico e literário. Há então, um retorno aos estudos que não se destina apenas a ideia curativa, mas também a manutenção do estado normal existente de indivíduos sadios. Surgem princípios básicos de exercícios 
para a conservação de um estado saudável já existente. No Renascimento há divisão entre os profissionais que deveriam reabilitar pessoas com alguma morbidade, e aqueles que cuidariam da saúde de pessoas sãs, e isso parece ter contribuído para que o objeto de trabalho da fisioterapia permanecesse direcionado ao atendimento de pessoas já enfermas. Teria sido essa a fase marcada pelo prelúdio da fisioterapia (DAVID, et al. 2013).

No período da industrialização, originada na Inglaterra, a atuação do profissional fisioterapeuta limita-se apenas a possibilidade de trabalho na reabilitação das condições que o organismo perdeu, excluindo modalidades de prevenção. As duas grandes guerras mundiais (5o primeiros anos do século XX), por consequência, causam um grande número de pessoas que necessitam de reabilitação, e com os recursos terapêuticos já utilizados até aquele momento, surgiu a fisioterapia como profissão (GAVA, 2004 apud COSTA, 20II).

\section{A profissão de fisioterapeuta}

As primeiras escolas surgem na Europa, precisamente na Alemanha (Kiel em 1902) e depois na Inglaterra. Na América do Norte a fisioterapia chega na Escola de Medicina de Harvard entre i912 e i916. A luta de um grupo de profissionais fez crescer a profissão no aspecto legal (CALVALCANTE et. al, 20II).

A profissão vislumbra uma atuação além da perspectiva reabilitadora, passando a atuar também nos pontos relacionados à elevação de saúde e prevenção dos insultos de saúde. A fisioterapia encarou grandes provocações para estabelecer a identidade profissional, não só no Brasil, mas também nos outros países da América e Europa. No início, a massagem foi utilizada como principal recurso terapêutico da fisioterapia, porém foi com o trabalho desenvolvido por meio da reabilitação física das pessoas que a profissão passou a ser reconhecida (ESPÍNDOLA e BORENSTEIN, 20II).

$\mathrm{Na}$ atualidade, a fisioterapia é definida como a ciência da saúde que estuda, previne e trata os distúrbios cinéticos funcionais, gerados por alterações genéticas, por traumas e por doenças adquiridas. Utilizando-se de conhecimento e recursos próprios como parte do processo terapêutico. O fisioterapeuta é o profissional da área da saúde que diminui ou previne as incapacidades físicas, por meios de recursos fisioterapêuticos conservadores, não-cirúrgicos (COFFITO 9). 
A atuação fisioterapêutica é de grande valia para a individualidade e a coletividade dos que usufruem desses serviços, contribuindo para melhoria da qualidade de vida e longevidade dos usuários, pois este profissional não atua somente quando a incapacidade já está instalada, mas também na área preventiva (MAIA et al., 2015).

A identidade do profissional fisioterapeuta foi construída permeada pelo objetivo de gerar a reabilitação física. Faz necessário destacar o papel do fisioterapeuta na saúde pública, atuação de maneira interdisciplinar, na elaboração e preparação de táticas para suprir às necessidades da comunidade em termos de prevenção de doenças e promoção de saúde, melhorando a qualidade de vida de todas as pessoas envolvidas nesse contexto social (ESPÍNDOLA e BORENSTEIN, 2OII).

A fisioterapia na reabilitação e na promoção de saúde, pela própria história da profissão, está ligada à melhora psicológica (aspectos psicológicos - transtornos de humor, e aspectos cognitivos - como a memória e a aprendizagem). A humanização da assistência fisioterapêutica significa reconhecer como sujeitos de direitos as pessoas que buscam nos serviços a resolução de suas necessidades (AUGUSTO, et.al, 20II).

Landmann et al. (2009) evidencia a importância do profissional fisioterapeuta em buscar através de sua visão global e de seus conhecimentos, facilitar a obtenção e aprimoramento de certas habilidades e conceitos necessários prévios, através de orientações e troca de experiências com outros profissionais, ou seja, atuando interdisciplinarmente.

David et al, (2013) afirma que é competência do profissional fisioterapeuta, atuar no planejamento, na programação, na coordenação, na execução e na supervisão da aplicação de métodos e técnicas que disponha. A promoção da saúde, em todos níveis de atenção, básica, secundária ou terciária, engloba o paciente em todos os aspectos (físicos, emocionais e culturais).

Para Oliveira (2015) na modernidade, a construção de uma abordagem dialógica para o nascimento de um novo discurso do profissionalismo na fisioterapia, pode ser uma possibilidade de quebradura com o modelo biomédico, o que levaria a compreensão do papel profissional no campo da saúde a partir de uma perspectiva crítica e reflexiva, como parte da autonomia e da identidade profissional. 


\section{A profissão de fisioterapeuta no Brasil}

No Brasil, antes da profissão de fisioterapeuta ser reconhecida, a utilização de técnicas fisioterapêuticas era realizada por médicos e auxiliares que tratavam as demandam dos necessitados. No ano de 1919, deu-se a fundação do Departamento de Eletricidade Médica da Faculdade de Medicina da USP, que tinha como foco o atendimento as sequelas da poliomielite (paralisia infantil) e de acidentes de trabalho. Assim, caracterizando, o profissional fisioterapeuta como somente reabilitador, sem a visão preventiva (VIEIRA, 2012 apud ARAÚJO, 2014).

Com as Leis, Decretos e Emendas foi conquistado a legalidade profissional, conferindo aos profissionais direitos e deveres. O quadro I mostra as principais Leis, Emendas e Decretos da fisioterapia.

Quadro r: Principais Leis, Emendas e Decretos que regem a profissão de Fisioterapeuta (ARAÚJO, 2014).

\begin{tabular}{|c|c|}
\hline 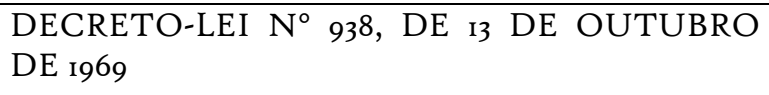 & $\begin{array}{l}\text { Provê sobre as profissões de fisioterapeuta e } \\
\text { terapeuta ocupacional, e dá outras providências. }\end{array}$ \\
\hline LEI N $^{\circ}$ 6.316, DE i7 DE DEZEMBRO DE 1975 & $\begin{array}{l}\text { Cria o Conselho Federal e os Conselhos Regionais de } \\
\text { Fisioterapia e Terapia Ocupacional e dá outras } \\
\text { providências. }\end{array}$ \\
\hline 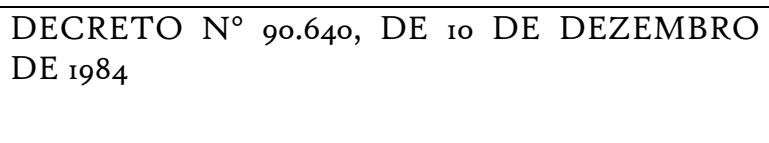 & $\begin{array}{l}\text { Inclui categoria funcional de Fisioterapeutas no } \\
\text { grupo de outras atividades de nível superior da Lei } \\
5.645 / 70 \text {. }\end{array}$ \\
\hline LEI N $^{\circ} .856$, DE Io DE MARÇO DE 1994 & $\begin{array}{l}\text { Fixa a Jornada de Trabalho dos Profissionais } \\
\text { Fisioterapeuta e Terapeuta Ocupacional. }\end{array}$ \\
\hline LEI № 9.098, DE 19 DE SETEMBRO DE 1995 & $\begin{array}{l}\text { Revoga as disposições que menciona, relativas a } \\
\text { recurso à instância ministerial. }\end{array}$ \\
\hline $\begin{array}{l}\text { EMENDA CONSTITUCIONAL № } 34, \text { DE } 13 \\
\text { DE DEZEMBRO DE 200I }\end{array}$ & $\begin{array}{l}\text { Dá nova redação à alínea c do inciso XVI do art. } 37 \\
\text { da Constituição Federal. }\end{array}$ \\
\hline LEI № 10.424, DE is DE ABRIL DE 2002 & $\begin{array}{l}\text { Acrescenta capítulo e artigo à Lei no } 8.080 \text {, de ig de } \\
\text { setembro de I99o, que dispõe sobre as condições para } \\
\text { a promoção, proteção e recuperação da saúde, a } \\
\text { organização e o funcionamento de serviços } \\
\text { correspondentes e dá outras providências, } \\
\text { regulamentando a assistência domiciliar no Sistema } \\
\text { Único de Saúde. }\end{array}$ \\
\hline
\end{tabular}


Fonte: COFFIITO, 2019.

\section{CONSIDERAÇÕES FINAIS}

Considera-se três subdivisões históricas que englobam todas as ciências da saúde: o período antes da primeira Guerra Mundial (que compreende a Antiguidade, Idade Média, o Renascimento e a Industrialização), o período das guerras e o período atual. Acontecimentos históricos sobre a utilização de recursos naturais para a recuperação de lesões mostram que desde os primórdios a fisioterapia foi utilizada baseada na experiência e na observação. No decorrer de sua história, houve fases de grandeza e de ostracismo.

A evolução das ciências da saúde mostra-se intimamente ligada aos períodos de fatos históricos, entretanto a profissão permaneceu durante muitos anos desconhecida, uma boa parcela da população ainda não conhece sua importância. De maneira global e por ter surgido em um cenário das ciências com função reabilitadora, colocou-se por muito

tempo os profissionais fisioterapeutas como exclusivamente da recuperação, deixando esses profissionais limitados a ações na saúde preventiva, por exemplo.

A Fisioterapia acompanhou as grandes mudanças e transformações do século XX, os profissionais que a exerciam souberam acrescentar descobertas novas e técnicas às suas práticas, elaborando e desenvolvendo uma ciência própria e um campo específico de atuação, independente das outras áreas da saúde.

A atuação do profissional fisioterapeuta vem caminhando no sentido de firmar-se no seu reconhecimento, sobretudo devido a sua utilização na prevenção e tratamento de doenças, pois este profissional não atua somente quando a deficiência ou incapacidade já está instalada, mas também na área preventiva, podendo atenuar diversas patologias, diminuindo o tempo de tratamento e consequentemente gastos. Com uma melhor legislação na prática fisioterapeuta, as transformações foram positivas, tanto no âmbito profissional, quanto no âmbito acadêmico.

\section{REFERÊNCIAS}


ARAÚJO, Aliceana Ramos Romão Menezes. Trajetória profissional do fisioterapeuta: reconhecimento e inter-relações no campo da saúde. Tese de doutorado. Universidade

Federal da Paraíba - UFPB. João Pessoa- PB; 2014. Disponível em: https://repositorio.ufpb.br/jspui/bitstream/tede/7330/I/arquivototal.pdf. Acesso em: I2 de julho de 202I.

AUGUSTO, Viviane Gontijo et. al. Promoção de saúde em unidades básicas: análise das representações sociais dos usuários sobre a atuação da fisioterapia. Ciência \& Saúde Coletiva, I6 (Supl. I):957-963, 2011.

CALVALCANTE, Cristiane de Carvalho Lima et. al. Evolução científica da fisioterapia em 40 anos de profissão. ISSN o103-5150. Revista Fisioterapia do Movimento, Curitiba, volume 24, n. 3, p. 513-522, julho/setembro. 2011.

CONSELHO FEDERAL DE FISIOTERAPIA E TERAPIA OCUPACIONAL (COFFITO). Resolução $\mathrm{n}^{\circ}$. 80, de 9 de maio de 1987. Disponível em: https://www.coffito.gov.br/nsite/?p=2838. Acesso em: I2 de julho de 202I.

CONSELHO REGIONAL DE FISIOTERAPIA E DE TERAPIA OCUPACIONAL (CREFITO 3). 20II. Disponível em: http://www.crefito3.org.br/dsn/fisioterapia.asp. Acesso em: I2 de julho de 202I

(CREFFITO 9). Disponível em: http:// www.crefitog.org.br/fisioterapia/o-que-efisioterapia/155. Acesso em: o9 de julho de 2021.

DAVID, Maria Laura Oliveira et al. Proposta de atuação da fisioterapia na saúde da criança e do adolescente: uma necessidade na atenção básica. Saúde em Debate. Rio de Janeiro, v. 37, n. 96, p. 120-I29, janeiro /março. 2013.

ESPÍNDOLA, Daniela Simoni; BORENSTEIN, Miriam Süsskind. Evolução histórica da fisioterapia: da massagem ao reconhecimento profissional (1894-2010). Fisioterapia Brasil, v. I2, n. 5, setembro/outubro de 2orr. 
FONSECA, João Pedro da. História da Fisioterapia em Portugal (Da origem a 1966). Instituto Politécnico de Lisboa. Escola Superior de Tecnologia da Saúde de Lisboa. Mestrado em Fisioterapia, 2012.

KLADNY, B. History of conservative spinal therapy. Unfallchirurg. v.II8, n.I. PMID 26542053. Doi: 10.1007/sool13-015-0098-4. Dezembro, 2015. Disponível em: https://link.springer.com/article/I0.1007/sooriz-015-0098-4. Acesso em: I2 de julho de 2021.

LANDMANN, Luciana Machado et. al. Espaço educacional e a possibilidade de atuação do fisioterapeuta. Ciência e cognição. V.I4 n.3; Rio de Janeiro novembro, 2009. Disponível em: http://pepsic.bvsalud.org/pdf/cc/vi4n3/vi4n3ao7.pdf. Acesso em: I2 de julho de 2021.

MAIA, Francisco Edison da Silva et al. A Importância da inclusão do profissional fisioterapeuta na atenção básica de saúde. Rev. Fac. Ciênc. Méd. Sorocaba, v. 17, n. 3, p. IIo - II5, 2015.

NASCIMENTO, Carolina Cristina do. Oficina de trabalho corporal em serviço de saúde mental. ıor f. Dissertação (Mestrado em Enfermagem Psiquiátrica) - Escola de Ribeirão Preto da Universidade de São Paulo, Ribeirão Preto, 2orI.

OliVeIRA, Ana Luiza de Oliveira. A Fisioterapia no estado de São Paulo: Um estudo sobre as representações dos profissionais. Tese de Doutorado. Universidade Estadual de Campinas. Faculdade de Ciências Médicas. Campinas, 2015.

TONELINI, Fabiana Morais. Atenção primaria: uma proposta da inserção das ações de fisioterapia. 2009. Iıo f. Dissertação (Mestrado em Saúde Coletiva) - Universidade Católica de SANTOS, Santos, 2009. 